\title{
Precompensation of gain nonuniformity in a Nd:glass amplifier using a programmable beam-shaping system
}

\author{
S. -W. Bahk, I. A. Begishev, and J. D. Zuegel \\ Laboratory for Laser Energetics \\ University of Rochester \\ 250 East River Road, Rochester, NY 14623 \\ Email: sbah@lle.rochester.edu \\ Phone: +1-585-273-4023, Fax: +1-585-273-1014
}

\begin{abstract}
A programmable liquid crystal beam-shaping system was installed for a 200-mJ optical parametric chirped-pulse amplification (OPCPA) system front end and was applied to dramatically improve the beam uniformity in the subsequent amplifier. A highly nonuniform beam profile caused by gain inhomogeneity in the amplifier was pre-compensated by the beam shaping system using significantly improved open-loop and closed-loop algorithms. The details of the improved algorithms will be described. The issues of running a liquid crystal device with a high-energy, ultrashort-pulse laser, such as damage risk and temporal contrast degradation, will be addressed.
\end{abstract}

Keywords: beam shaping, spatial light modulator, laser amplifier, optical parametric chirped pulse amplification, Nd:glass, high energy laser

\section{Introduction}

A dynamic beam-shaping system using a liquid crystal spatial light modulator (SLM) underwent a successful transition from the initial proof-of-principle demonstration $[1,2]$ to an implementation in a high-energy laser such as the OMEGA EP at the University of Rochester [3]. The operation principle of the beam-shaping system is based on the phase-only carrier method, which enables one to have arbitrary two-dimensional (2-D) control of both the laser-beam fluence and wavefront by adjusting the modulation depth and the bias of the carrier phase. A liquid crystal on silicon (LCOS) SLM can be used to create a programmable high-frequency carrier phase. Closed-loop operation of such a device paired with feedback from a near-field camera or a wavefront sensor dramatically improves the performance [2].

Gain precompensation [4] and spot shadowing [5, 6] are important applications of a dynamic beam shaper in high-energy lasers. The precompensation of gain inhomogeneity in amplifiers reduces the peak-to-mode in fluence distribution in such a way that the total energy of the beam can be increased without risking damage to the optics. The gain precompensation in OMEGA EP long-pulse (ns) beamlines is achieved in multiple steps using both static and dynamic beam shapers [3, 7]. The dynamic beam shaper is located in the low-energy front end, where a residual 2-D correction map is applied to improve the overall system gain precompensation performance and the uniformity of the final output beam. A similar gain precompensation experiment was performed in another facility for a $\mathrm{Nd}$ :glass amplifier but with insignificant improvement in beam uniformity [8].

In this paper we present a more-challenging application of dynamic beam shaping in the context of a 50-J, 700-fs optical parametric chirped-pulse amplification (OPCPA) system. The high degree of gain saturation in the optical parametric amplification (OPA) crystals precludes the possibility of installing the SLM before the OPA. The incident beam energy on an SLM installed after the OPA is high enough, however, to damage the device if not carefully managed. Pulse contrast degradation caused by the secondary reflection from the front surface of the SLM cover glass is a non-negligible problem in this pulse-width regime. It introduces a prepulse 30-ps before the main pulse. We have been able to mitigate or remove these problems. The details of our approach will be described later.

Significant improvements and diversifications in the algorithms and the mode of operations of the carrier method have been made. First, a large discrepancy was found between the analytic transmission function derived in [1] and the experimental function. An empirical formula is introduced here that agrees better with the measurements. It greatly improves the accuracy of the open-loop algorithm. The closed-loop algorithm introduced in [2] is based on incrementing or decrementing a fixed unit-step size of the digital command map. It can be inefficient to use a fixed step size where many steps are required for convergence. A more-efficient closed-loop algorithm will be discussed.

The type of carriers used in the carrier beam-shaping method is not limited to one-dimensional (1-D) rectangular carrier. A checkerboard-pattern carrier was successfully used in the lasercavity mode shaping in [9]. The choice of the transmitted diffrac- 
tion order does not need to be the zeroth order. A sawtoothshaped carrier beam can maximize the diffracted energy into the first-order diffraction term, where the first-order term is chosen to be the main transmitted beam. Such mode of operation is fail-safe since the beam can propagate only in the presence of a carrier.

The improvements in the carrier beam-shaping algorithms will be discussed followed by detailed descriptions of the laser system and the experimental results.

\section{Improvement and diversification of the original carrier beam shaping method}

In the original phase-only carrier beam-shaping method, a rectangular carrier phase is applied to an SLM. A beam incident on the SLM acquires high-frequency modulation in phase and diffracts into the zeroth- and the first-order diffractions. As the modulation peak-to-valley ( $\mathrm{p}-\mathrm{v}$ ) approaches $\pi$, more energy diffracts into the first- and higher-order diffractions. The diffracted beams are filtered and only the zeroth-order beam is allowed to pass. In this way the intensity transmission factor can be adjusted by controlling the modulation depth of the carrier phase. A 2-D transmission map as a function of carrier amplitude can be generated to achieve a desired beam shape. This principle can be modified in such a way that the firstorder diffraction beam passes whereas the zeroth-order beam is blocked. The benefit of such a configuration is that the beam does not propagate when the SLM fails to introduce the carrier, which is a useful feature for fail-safe operation in a high-energy laser. The disadvantage is that the maximum transmission cannot exceed $50 \%$ because the first-order diffractions are equally split. The low efficiency can be improved by using a sawtooth carrier phase (blazed grating) instead of a rectangular one. The diffraction efficiency of the first-order diffraction for the sawtooth carrier phase can be very high. These two modes of operation are schematically shown in Fig. 1, where Fig. 1(a) describes the original carrier beam-shaping setup and Fig. 1(b) shows the modified carrier method in Littrow configuration. We refer to the first configuration as normal mode and the second as diffractive mode. For later discussions, the transmission $[T(x, y)]$ of a carrier method in the near field of each setup after filtering is defined as

$$
T(x, y)=\frac{E_{0 \text { th order }}(x, y)}{E_{\text {inc }}(x, y)},
$$

and

$$
T(x, y)=\frac{E_{1 \mathrm{st} \text { order }}(x, y)}{E_{\text {inc }}(x, y)},
$$

for normal-, and diffractive-mode methods, respectively. $E_{\mathrm{inc}}$ is the incident local energy on the SLM and $E_{0 \text { th order }}, E_{1 \text { st order }}$ are the local reflected energy contained in the zeroth- or firstorder diffraction beam, respectively.

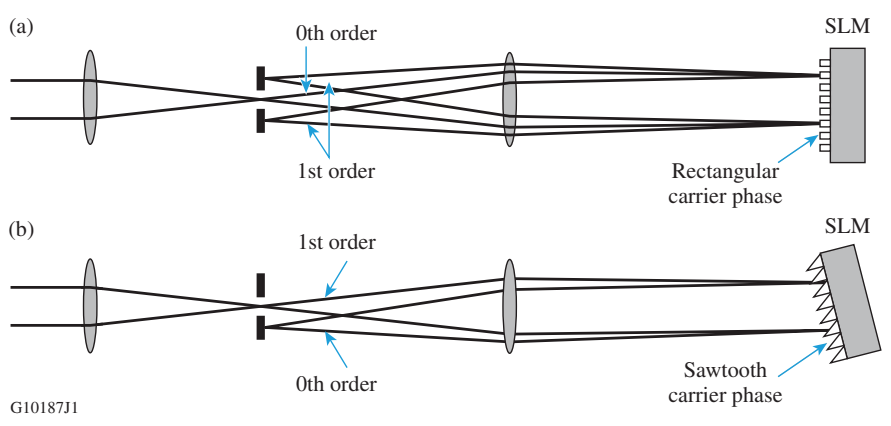

Figure 1: Two modes of carrier beam shaping. (a) Normal mode using zerothorder beam as the main beam. (b) Diffractive mode using the first-order beam as the main beam.

\subsection{Improvement in open-loop algorithm}

The algorithm described here assumes using an LCOS-SLM device whose voltage-to-phase-retardation response is linearized by using a look-up-table (LUT). Use of an LUT does not completely remove the small variations in phase retardation in individual pixels of an SLM. In our case, the variation is $\pm 6 \%$. In an open-loop algorithm, we attempted to achieve the best beamshaping performance in a single step, neglecting these small variations. We used a reflective LCOS-SLM from Hamamatsu (X10468-03). The phase of an individual pixel is controlled by applying voltage on the nematic liquid crystal sandwiched between two parallel-aligned alignment layers. The SLM has $600 \times 80020-\mu \mathrm{m}$ pixels over $12-\times 16-\mathrm{mm}^{2}$ area. The phase retardation from 0 to 2 waves can be independently introduced on each pixel.

\subsubsection{Normal mode}

The theoretical normal-mode transmission for a rectangular carrier phase with $\pm 2 \pi A$ fluctuation was shown to be $|\cos (2 \pi A)|^{2}$ in [1]. The actual transmission deviates, however, from the theoretical prediction. The theoretical and measured transmission curves are shown in Fig. 2. The transmission loss from the SLM's reflectivity (93\%) was not included in the calculation or measurements. The line of blue circles is the theoretical prediction, whereas the colored solid lines are the transmissions measured at three different carrier frequencies by varying the carrier amplitude $(A)$ from 0 to 1 wave. The transmission at each carrier frequency was averaged over nine different points on the SLM. The black, blue, and red solid lines correspond to the carrier periodicity of two, four, and six-pixels, respectively. The measured transmission curves show discrepancies with the theoretical curve in that the location of the first minimum is further away from the theoretical 0.25 and the second peak is lower than 1. Another theoretical transmission curve based on a sinusoidal carrier is shown by the line of purple circles. The analytic transmission function of a sinusoidal carrier is a Bessel function $\left(J_{0}\right)$. Comparison of the analytic and measured transmission curves shows that the measured transmission has characteristics somewhere between the rectangular and sinusoidal carriers. The deviation from the rectangular-carrier transmission curve 
becomes larger as the carrier frequency increases. This suggests that the corners of the actual carrier wave is rounded and this effect becomes more pronounced at high frequency. Based on this observation, the empirical transmission function can be expressed using both cosine and Bessel functions as follows:

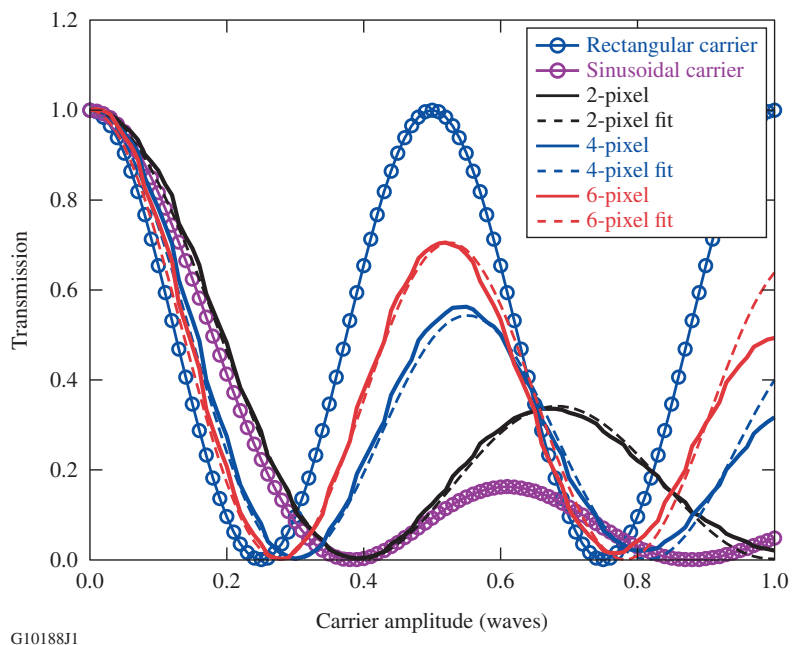

Figure 2: (Color online) Transmission curve with respect to varying wavefront amplitude. The six-pixel-period carrier transmission is closer to that of the rectangular carrier and the two-pixel-carrier transmission is closer to the sinusoidal carrier transmission. There is a finite extinction due to scattering.

$$
T(A)=(1-c)\left[(1-a) \cos (2 \pi b A)+a J_{0}(2 \pi b A)\right]^{2}+c,
$$

where $T$ is the transmission as a function of carrier amplitude $A$. The fit parameters $(a, b, c)$ for each averaged transmission curve are given in Table 1 . The numerically fit transmission functions are shown as dashed lines in Fig. 2.

Table 1: Rectangular-carrier transmission parameters. The transmission of the two-pixel (px)-period carrier is fit over $0<A<1$. The fit range for four-pixeland six-pixel-period carriers is $0<A<0.6$.

\begin{tabular}{ccccc}
\hline carrier period & $a$ & $b$ & $c$ & $A_{\min }$ \\
\hline 2-px period & 0.64 & 0.80 & 0.0015 & 0.40 \\
4-px period & 0.39 & 0.95 & 0.0006 & 0.30 \\
6-px period & 0.23 & 0.97 & 0.0001 & 0.28 \\
\hline
\end{tabular}

The locations of the first minima calculated from the analytic function with the given fit parameters are shown in the $A_{\min }$ column. The minimum transmission is the same as $c$. The value of $c$ is shown to the fourth digit to emphasize the fact that there is a low level of leak even at "zero" transmission. The extinction is better at a lower carrier frequency.

The choice of carrier frequency depends on beam-shaping applications. The six-pixel-period carrier requires smaller carrier wavefront amplitude than the two-pixel carrier ( 0.28 versus 0.40 ) to achieve maximum contrast, leaving the rest of the dynamic range for wavefront correction. On the other hand, the two-pixel-period carrier allows more digital levels per unit transmission change, therefore a finer beam-shaping scale.

\subsubsection{Diffractive mode}

The transmission characteristic of the diffractive mode beam shaping using a sawtooth carrier is shown in Fig. 3. The transmission with respect to the blazed-grating amplitude was measured at different grating periods. Each curve was averaged over nine different points on the SLM. The analytic transmission function for a sawtooth carrier is

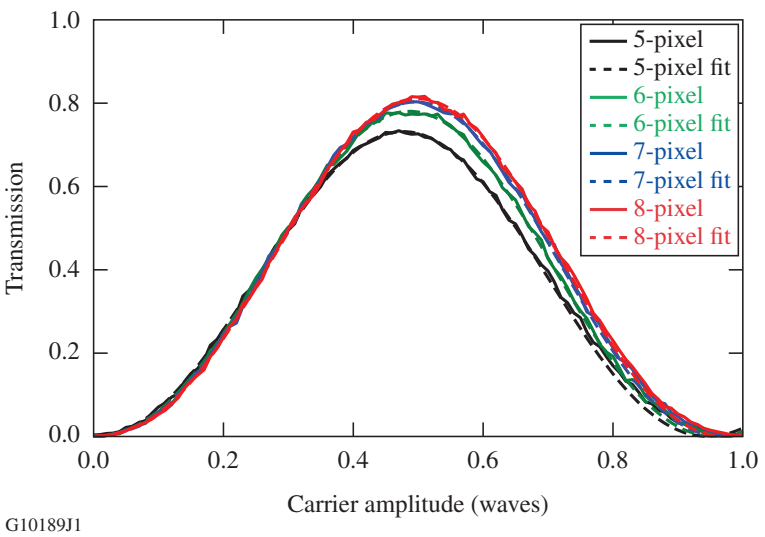

Figure 3: (Color online) Transmission characteristic of diffractive mode carrier.

$$
T(A)=\Gamma\left\{\frac{\sin [2 \pi(A-0.5)]}{2 \pi(A-0.5)}\right\}^{2},
$$

where $\Gamma$ is the diffraction efficiency and $2 A$ is the $p-v$ of the sawtooth carrier. $\Gamma$ is lower than 1 because the sawtooth shape is pixelated in LCOS-SLM. As in the case of the rectangular carrier, the analytic transmission function does not agree well with the measured transmission, albeit the discrepancy is smaller in this case. We find that the following empirical expression, made of trigonometric and Bessel functions similar to Eq. (3), fits the data better than the analytic function:

$$
T(A)=c\left[(1-a) \sin (2 \pi b A)+a J_{2}(2 \pi b A)\right]^{2} .
$$

The fit parameters $(a, b, c)$ are summarized in Table 2 .

Table 2: Sawtooth-carrier transmission parameters. The curves were fit over $0<A<0.7$.

\begin{tabular}{cccccc}
\hline carrier period & $a$ & $b$ & $c$ & $A_{\max }$ & $T_{\max }$ \\
\hline 5-px period & 0.52 & 0.61 & 1.88 & 0.48 & 0.73 \\
6-px period & 0.65 & 0.65 & 2.60 & 0.49 & 0.78 \\
7-px period & 0.69 & 0.66 & 2.89 & 0.50 & 0.80 \\
8-px period & 0.70 & 0.67 & 3.00 & 0.50 & 0.81 \\
\hline
\end{tabular}

Retaining a high diffraction efficiency is important for a diffractive mode. Figure 3 suggests that the maximum transmission reaches $81 \%$ with an eight-pixel carrier but does not improve much beyond that. The carrier frequency cannot be set 
too low in any case because the first- and zeroth-order diffractions must be angularly separated. The effective spatial resolution is also reduced with a large period carrier. As in the rectangular carrier setup, the $93 \%$ reflectivity of the device was not included in the measurement. The actual maximum throughput is $75 \%$ for the eight-pixel carrier.

The fit parameters in either in normal or diffractive mode must be recharacterized whenever there is a change in the design of the LCOS chip, such as the fill factor.

\subsection{Closed-loop algorithm}

Based on the quasi-linear response of the SLM to command voltage, a general form of a 2-D command map $C(x, y)$ including wavefront shaping $[W(x, y)$, bias of carrier] and transmission control $[A(x, y)$, envelope of carrier, same as $A$ in Eqs. (3), (5)] can be written as,

$$
\begin{aligned}
C(x, y) & =\gamma[W(x, y)+A(x, y) \times(\text { carrier })], \\
& =C_{W}(x, y)+C_{A}(x, y) \times(\text { carrier }),
\end{aligned}
$$

where the spatial frequency of $W(x, y)$ should be smaller than that of the carrier. $\gamma$ is a conversion factor from wavefront unit to command voltage, and $C_{W}$ and $C_{A}$ are command maps corresponding to $W$ and $A$, respectively. Because of the small local variations in the phase retardation at a constant voltage across the device, $C(x, y)$ producing the required $W(x, y)$ and $A(x, y)$ cannot be exactly determined in a single step. A closed-loop algorithm is required to improve the result. A variant of Newton method and a closed-loop algorithm based on that method will be discussed.

\subsubsection{Constant maximum-derivative Newton method}

The Newton method iteratively finds a solution for the equation $F(x)=0$ using

$$
x_{n+1}=x_{n}-\frac{F\left(x_{n}\right)}{F^{\prime}\left(x_{n}\right)},
$$

where $x$ corresponds to the command map state. For given wavefront and transmission objectives ( $W_{\mathrm{obj}}$ and $\left.T_{\mathrm{obj}}\right)$, the function $F(x)$ corresponds to $W(x)-W_{\mathrm{obj}}$ for wavefront shaping and $T(x)-T_{\text {obj }}$ for intensity shaping. $W(x)$ represents the actual wavefront produced by the SLM at a given command voltage $x=C_{W}$, and $T(x)$ represents the actual transmission of intensity at a given command voltage $x=C_{A}$. With only the approximate form of $W(x)$ or $T(x)$ known, the derivative in the the Newton search cannot be calculated exactly. $F^{\prime}(x)$ can be replaced with the derivative of an analytic expression for the mean response. This approach works quite well most of the time but it is not stable. Toward the end of the iterations, $x_{n}$ can be caught up in an oscillation around the solution and might even diverge especially where the response is jagged. Our approach is to fix the derivative to a constant value smaller or larger than the actual derivative, depending on its sign. This ensures the convergence of the iterator for any noisy functions. The original Newton search method is modified to

$$
x_{n+1}=x_{n}-\frac{F\left(x_{n}\right)}{F_{\max }^{\prime}},
$$

where $F_{\max }^{\prime}$ is smaller than $\inf \left[F^{\prime}(x)\right]$ for a noisy downhill function or larger than $\sup \left[F^{\prime}(x)\right]$ for a noisy uphill function. We present a graphical illustration of this approach in Fig. 4 for a noisy uphill function. The initial solution starting from the right-hand side approaches toward the solution without divergence using a positive constant derivative larger than any part of the actual derivative in the function even in noisy condition. A starting point located in the left-hand side again approaches toward the solution without divergence. A similar argument can be given for a noisy downhill function. Figure 5 shows the actual derivatives of the measured normal-mode transmission function of the six-pixel carrier at various points on the SLM which is very noisy. Since the transmission function for the normal beam-shaping mode is globally downhill, the appropriate constant derivative can be set to -10 lower than any measured derivative.

The proposed method is different from the damped Newton method, which is

$$
x_{n+1}=x_{n}-\alpha \frac{F\left(x_{n}\right)}{F^{\prime}\left(x_{n}\right)},
$$

where $\alpha$ is a damping factor. The damping factor $\alpha$ in Eq. (10) allows one to make a conservative move in the next step, but it is not free from the erratic behavior of $F^{\prime}(x)$. It is also unstable where $F^{\prime}(x)$ is close to zero, which is one of the general problems of the Newton method. On the other hand, the constant maximum-derivative Newton method always converges. The proposed method is well suited for the case when the general behavior of a function can be expressed as an invertible analytic function but its micro-behavior is not well characterized. The proximity of the solution is reached by inverting the analytic function such as Eq. (3) or Eq. (5). The final solution is refined by Eq. (9).

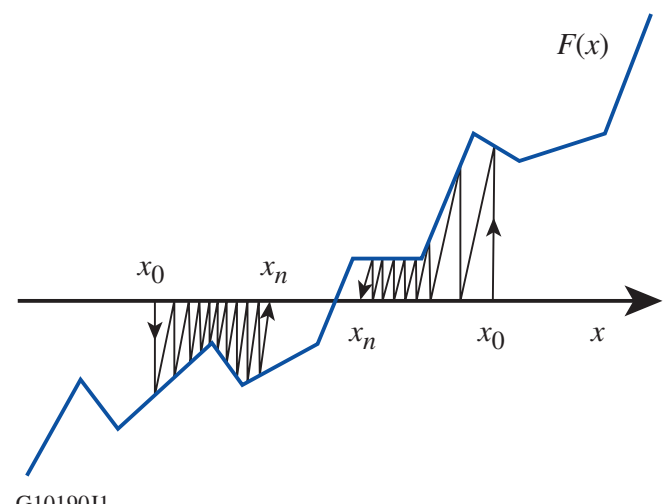

Figure 4: Illustration of constant-maximum-derivative Newton search algorithm. A zero of a noisy uphill function is found without diverging by using a constant search derivative larger than $\sup \left[F^{\prime}(x)\right]$, even with local downhill sections.

\subsubsection{Wavefront and fluence shaping algorithms}

The iteration algorithms for either wavefront shaping or fluence shaping are formulated using the constant maximum-derivative Newton method. Since $x_{n}$ in Eq. (9) represents an independent point in a 2-D command map, $F_{\text {max }}^{\prime}$ can be independently 


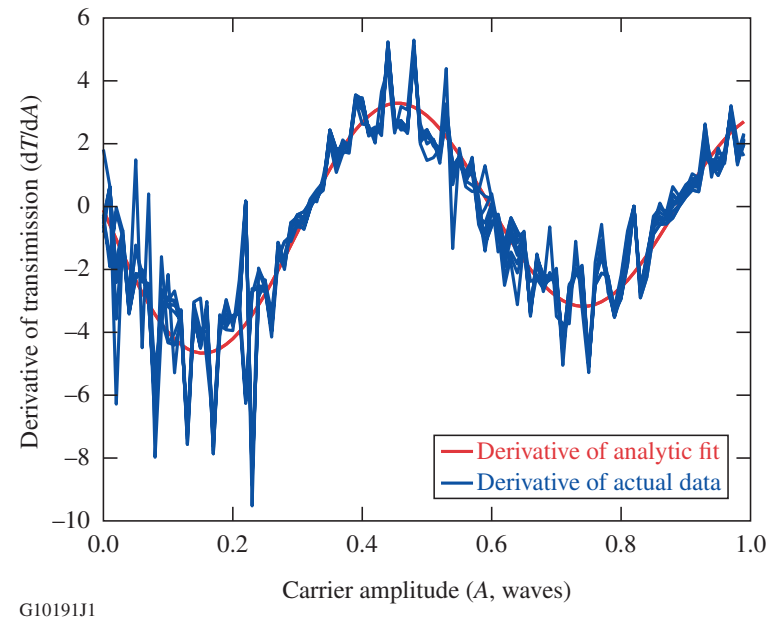

Figure 5: (Color online) The derivative of transmission function. The actual derivative of the measured transmission data of six-pixel period carrier modulation is shown on top of the derivative of the empirical formula.

set for different points on the SLM. Nonthelss, a single number is used nonetheless for the entire 2-D map for convenience. Since the wavefront response with respect to its command voltage $C_{W}$ is a monotonically increasing function, one chooses $F_{\max }^{\prime}>\sup \left[F^{\prime}(x)\right]$. The wavefront response function can be expressed as

$$
W\left(C_{W}\right)=W_{0}\left(C_{W}\right)+\eta\left(C_{W}\right),
$$

where $W_{0}\left(C_{W}\right)$ represents an averaged response of the SLM with respect to the command map. In general this function is a linear function of $C_{W}$, and $\eta\left(C_{W}\right)$ accounts for the small deviation in the actual response from the ideal linear function. With the objective wavefront denoted as $W_{\text {obj }}$ and the constant search derivative as $\left(d W / d C_{W}\right)_{\max }$, the iteration process of the command voltage can be established as follows:

$$
\begin{aligned}
C_{W, 1} & =W_{0}^{-1}\left(W_{\mathrm{obj}}\right), \\
C_{W, n+1} & =C_{W, n}-\frac{1}{\left(d W / d C_{W}\right)_{\max }}\left[W\left(C_{W, n}\right)-W_{\mathrm{obj}}\right] .
\end{aligned}
$$

The function $W\left(C_{W, n}\right)$ implies the measurement of the actual wavefront with the $n$th command map state, $C_{W, n}$.

An intensity-shaping closed-loop algorithm can be similarly constructed. The transmission function is again expressed as the sum of an averaged response and small deviation as

$$
T\left(C_{A}\right)=T_{0}\left(C_{A}\right)+\zeta\left(C_{A}\right),
$$

where $T_{0}\left(C_{A}\right)$ denotes either Eq. (3) or Eq. (5) and $\zeta\left(C_{A}\right)$ is a small deviation function. Defining the objective transmission as $T_{\text {obj }}$ and the maximum derivative as $\left(d T / d C_{A}\right)_{\max }$, the iteration process for the carrier envelope command voltage is

$$
\begin{aligned}
C_{A, 1} & =T_{0}^{-1}\left(T_{\mathrm{obj}}\right), \\
C_{A, n+1} & =C_{A, n}-\frac{1}{\left(d T / d C_{A}\right)_{\max }}\left[T\left(C_{A, n}\right)-T_{\mathrm{obj}}\right] .
\end{aligned}
$$

The constant search derivative $\left(d T / d C_{A}\right)_{\max }$ is to be understood as a negative value smaller than the infimum of $d T / d C_{A}$ in the case of normal mode beam shaping or a positive value larger than the supremum of $d T / d C_{A}$ in the case of reverse beam shaping. In either case the valid interval of $C_{A}$ is from zero to $C_{A, \min }$ or $C_{A, \max } . C_{A, \min }$ and $C_{A, \max }$ are command voltages corresponding to $A_{\min }$ or $A_{\max }$ shown in Table 1 and Table 2 . The inversion function $T_{0}^{-1}$ is numerically calculated.

Since the fluence is the quantity measured directly, it is more useful to express the above iteration formula in terms of fluences. Since the objective fluence map, $F_{\text {obj }}$, is $T_{\text {obj }} \times F_{\text {init }}$ where $F_{\text {init }}$ is the initial fluence map,

$$
\begin{aligned}
C_{A, 1} & =T_{0}^{-1}\left(F_{\text {obj }} / F_{\text {init }}\right), \\
C_{A, n+1} & =C_{A, n}-\frac{1}{\left(d T / d C_{A}\right)_{\max }}\left[F\left(C_{A, n}\right)-F_{\text {obj }}\right] / F_{\text {init }} .
\end{aligned}
$$

The iteration does not start if $\left|F_{\text {init }}\right|<\epsilon$ or $\left|F_{\text {init }}-F_{\text {obj }}\right|<\epsilon$ since it suggests that there is either no beam to shape or there is no need to shape. $C_{A}$ locally corresponding to these conditions is fixed at 0 for normal mode or at $C_{A \text {, max }}$ for diffractive mode. The iteration stops if $\left|F\left(C_{A, n}\right)-F_{\text {obj }}\right|<\epsilon$ or if $C_{A, n}>C_{A, \text { min }}$ for normal mode or if $C_{A, n}<0$ for diffractive mode. The second criterion is needed when $F_{\text {obj }}$ is specified to be much smaller than the system can handle. For example, the minimum transmission of a rectangular carrier can be a few percent high for a certain LCOS-SLM model with a low fill factor, while the objective transmission can be ideally set to $0 \%$. Unable to reach the solution, the iteration will keep increasing $C_{A, n}$ past the minimum (same as $A_{\min }$ in Table 1). Setting an upper limit to $C_{A, n}$ will prevent this runaway situation.

Equations (12) and (15) can be rewritten using gain parameters $\left(g_{W}\right.$ and $\left.g_{A}\right)$ that are positive and smaller than 1 :

$$
C_{W, n+1}=C_{W, n}+g_{W}\left[W_{\mathrm{obj}}-W\left(C_{W, n}\right)\right],
$$

and

$$
C_{A, n+1}=C_{A, n} \pm g_{A}\left[F\left(C_{A, n}\right)-F_{\text {obj }}\right] / F_{\text {init }},
$$

where positive and negative signs in front of $g_{A}$ correspond to normal and diffractive modes, respectively.

\section{Application in gain precompensation for a Nd:glass am- plifier}

The multi-terawatt (MTW) laser at the Laboratory for Laser Energetics of the University of Rochester consists of an OPCPA front end and two glass amplifiers[10]. The OPCPA front end is made of three OPA stages, one before, and the other two after the stretcher. The preamplification before the stretcher improves the pulse contrast [11]. The two OPA stages after the stretcher amplifies the pulse up to $\sim 200 \mathrm{~mJ}$. The first main amplifier after the OPCPA front end is a 25-mm-diam Nd:glass rod amplifier (RA) in a double-pass configuration. The second main amplifier is made of four $400-\mathrm{mm}$ aperture disks installed in zig-zag formation at the Brewster angle. The 200-mJ pulse from the OPCPA front end is amplified to $2 \mathrm{~J}$ through the rod 
(a)

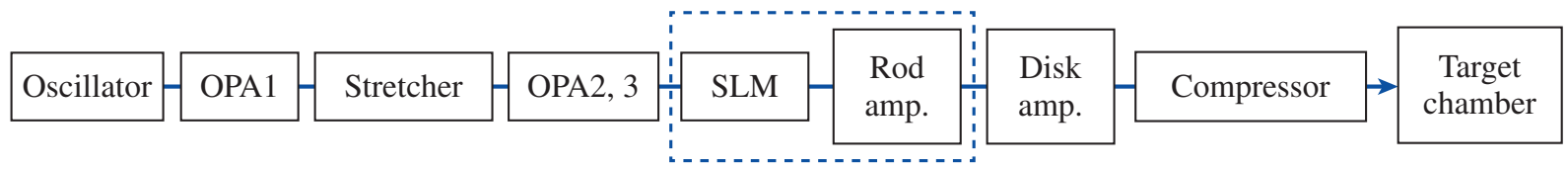

(b)

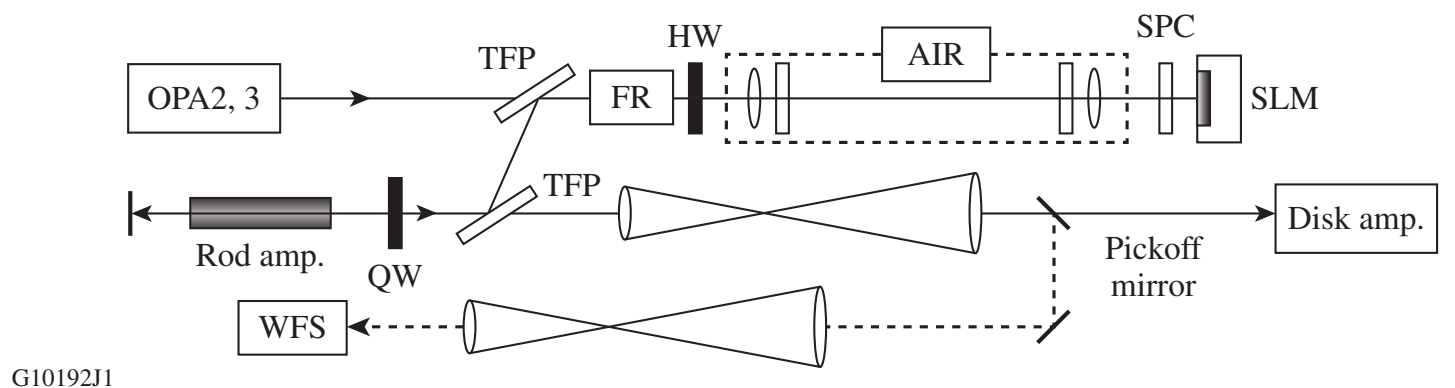

Figure 6: (a) Overall schematic of the Multi-Terawatt (MTW) laser; (b) detailed schematic of the MTW beam-shaping system. The image relay shown between the rod amplifier and disk amplifier is a simplified representation. TFP: thin-film polarizer, FR: Faraday rotator, HW: half-wave plate, QW: quarter-wave plate, SPC: static phase corrector, WFS: wavefront sensor.

amplifier and can be boosted up to $120 \mathrm{~J}$ by four passes in the disk amplifier. The amplified pulse is then compressed and sent to the target chamber. The schematic of the whole system is shown in Fig. 6(a). The spatial light modulator (SLM) is placed between the OPCPA front end output and the rod amplifier at an image plane. A more-detailed schematic of this part of the system is shown in Fig. 6(b). The square output beam from the OPCPA front end is anamorphically imaged to the rectangular area of the SLM (see $\S 2.1$ ). It is restored back to the original square beam by double passing the same anamorphic imaging system. A static phase corrector in front of the SLM cancels the intrinsic wavefront error in the SLM [2]. The beam is shaped to a gain-precompensated profile by the SLM using the normalmode carrier method. After the SLM, the beam is switched out by polarization optics and imaged to the exit surface of the rod amplifier. On double passing the rod amplifier, the amplified pulse is magnified and imaged to the disk amplifier. A sample of the injection beam to the disk amplifier is picked off by an uncoated mirror blank and imaged to a wavefront sensor.

The anamorphic image relay (AIR) is made of two pairs of cylindrical lenses. The outer and inner pair of cylindrical lenses separately images the horizontal and vertical dimensions with different magnifications. The $13-\times 13-\mathrm{mm}^{2}$ input beam is transformed into a $12-\times 16-\mathrm{mm}^{2}$ beam on the SLM. An AIR works for only a fixed object distance. The error in the object distance is recovered by adjusting the image distance, but only in one dimension in AIR. The tolerance in object positioning within which the imaging conditions in horizontal and vertical dimensions remain same can be derived by using the Maréchal criterion for the defocus term as

$$
\Delta z_{\mathrm{obj}} \leq \frac{D^{2}}{\lambda \Delta M^{2}},
$$

where $\Delta M^{2}=\left|M_{x}^{2}-M_{y}^{2}\right|$ and $D$ is the smallest feature size of the object. With a few-hundred-micron feature size in the beam and $\Delta M^{2}=0.66$, the positioning tolerance is tens of millimeters.

The full utilization of the rectangular area of the SLM reduces the peak fluence by $25 \%$ and allows for a safer operation below the damage threshold of the SLM. The damage threshold of LCOS samples, which were the same kind as used in the device, was found to be $230 \mathrm{~mJ} / \mathrm{cm}^{2}$ using the same 2.5-ns OPCPA pulses running at $5 \mathrm{~Hz}$. The peak fluence of the anamorphically imaged beam on the SLM at the maximum OPCPA energy $(200 \mathrm{~mJ})$ does not exceed $160 \mathrm{~mJ} / \mathrm{cm}^{2}$. A vacuum system is not needed for the AIR; there is a large separation between the sagittal and tangential focal planes, significantly reducing the intensity near the focus.

The reflection from the AR-coated first surface of the SLM cover glass introduces a prepulse 30 ps before the main pulse, down at the $-40-\mathrm{dB}$ level. This is not acceptable for experiments requiring a high-contrast pulse. The SLM was customized with a wedged cover glass to eliminate the prepulse. The wedge angle is $4.1 \mathrm{mrad}$. An alternative would be diffractive-mode beam shaping, where the prepulse from the cover glass is automatically separated from the main pulse.

The beam profile at the diagnostic wavefront sensor [WFS in Fig. 6(b)] before and after the amplification in the rod amplifier is shown in Fig. 7. The relatively uniform input OPCPA beam profile shown in Fig. 7(a) is highly distorted at the bottom corners after amplification in the rod amplifier [Fig. 7(b)].

Based on the two fluence maps measured before and after the amplification, a gain-precompensated fluence map, or an objective map, can be designed that will become uniform on amplification. Assuming the objective is a super-Gaussian beam the objective fluence has the following form

$$
F_{\text {obj }}=F_{\text {peak }} \times F_{0} \times T_{\text {gain }},
$$

where $F_{\text {peak }}$ is a constant that adjusts the maximum objective 
(a)

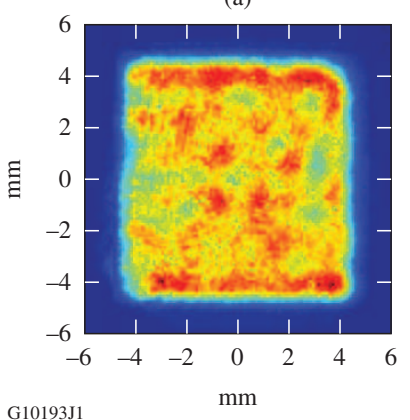

(b)

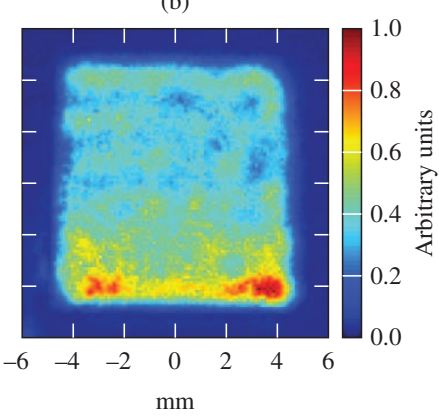

Figure 7: (Color online) Beam profile (a) before and (b) after amplification through the rod amplifier. The lower bottom corners of the beam become hot after amplification. The dimensions of the images correspond to camera space in the diagnostic beam path.

fluence and $F_{0}$ is a super-Gaussian beam profile usually expressed as

$$
F_{0}=\exp \left\{-\left[\left(\frac{x-x_{c}}{R_{x}}\right)^{m}+\left(\frac{y-y_{c}}{R_{y}}\right)^{m}\right]\right\} .
$$

$T_{\text {gain }}$ is a map of the ratio between the fluences before and after amplification:

$$
T_{\text {gain }}=\frac{F_{\text {init }}}{F_{\text {amp }}},
$$

where $F_{\text {init }}$ and $F_{\text {amp }}$ correspond to Fig. 7(a) and (b), respectively.

The ratio map calculated as above cannot be directly used because it is not well defined where the denominator is close to or equal to zero. A special region of interest is created to avoid this problem and to add stability in a 2-D polynomial fit. The region of interest consists of an interior region and an exterior rectangular frame as shown in Fig. 8. The interior region $\left(\Omega_{A}\right)$ is where the signal level of both $F_{\text {init }}$ and $F_{\text {amp }}$ is above $5 \%$ of the maximum signal level. The outer region $\left(\Omega_{B}\right)$ is a region of the rectangular frame. The coefficients for a set of 2-D Legendre polynomials are calculated to fit the measured gain ratio within the interior region and all zeros in the exterior frame region. The zeros in the frame stabilize the edge of the polynomials. The outer region has dimensions of $2 x_{0} \times 2 y_{0}$ with the frame thickness $d R$. There is a no strict rule setting $x_{0}, y_{0}$, and $d R$. The frame size should be large enough to cover the interior region but not bigger than the camera area; $d R$ should be set thin enough to provide the best numerical fit. The number of modes used in this case was 240 . The polynomial representation of $T_{\text {gain }}$ is normalized to 1 at the end.

The polynomial fit in combination with the super-Gaussian envelope $F_{0}$ provides a smooth objective profile that can be experimentally achieved. In Eq. (19) $F_{\text {peak }}$ is a single number that can be adjusted from zero to a few times the maximum of the $F_{\text {init }} ; F_{\text {peak }}$ is set as high as possible to minimize the energy loss from beam shaping.

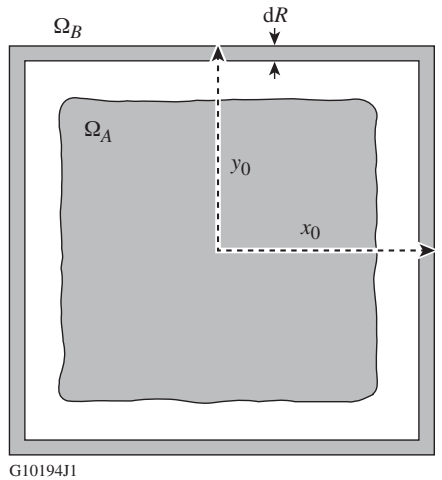

Figure 8: Region of interest for polynomial fit.

As a final step, the part of the objective map accidentally set higher than $F_{\text {init }}$ is set to $F_{\text {init }}$ and set to zero where it is negative. The remaining sharp features are low-pass filtered.

Figure 9(a) shows the beam shaping result precompensating the gain nonuniformity in the amplifier shown in Fig. 7(b). The precompensated beam is restored to a flat beam profile after amplification as shown in Fig. 9(b). It takes only half an hour from the measurement of an uncompensated rod amplifier beam to a design of an objective map and to a completion of the closedloop. The beam shape is maintained at near optimum condition in daily operations by rerunning the closed-loop.

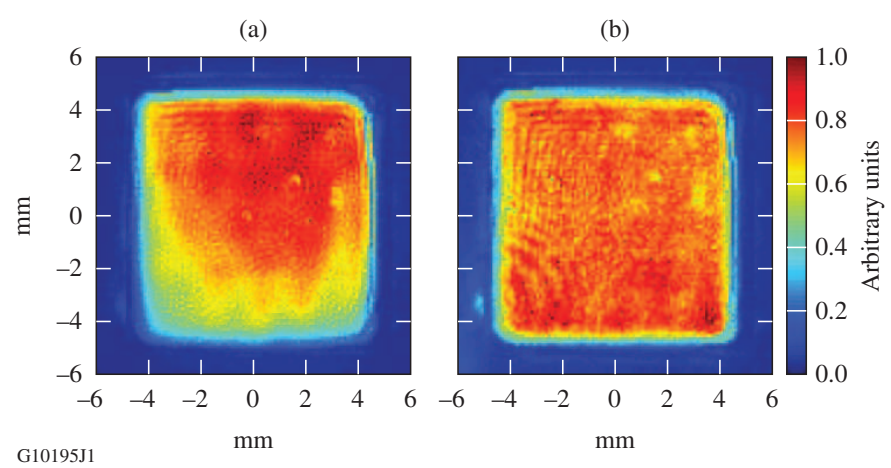

Figure 9: (Color online) Precompensated beam profile (a) before and (b) after amplification through the rod amplifier. The lower bottom corners of the beam is attenuated before amplification and becomes even after amplification.

The beam uniformity achieved in the rod amplifier stage is not degraded as the beam passes through the next amplifier, which is the disk amplifier. The profile of a 39-J beam at the output of the disk amplifier near an image plane is shown in Fig. 10. The beam contrast as defined in the Eq. (1) of [12] is $9 \%$. Improvement in the beam uniformity helps to safely operate the system under safe condition by keeping the maximum fluence below the damage threshold of the compressor gratings. The maximum fluence of this beam is $1.1 \mathrm{~J} / \mathrm{cm}^{2}$ whereas the damage threshold of the grating is $1.5 \mathrm{~J} / \mathrm{cm}^{2}$ at $10 \mathrm{ps}$ measured by $\mathrm{N}$-on- 1 tests.

We have not seen any pulse compressibility issue coming from the use of SLM for the MTW laser. The amplified pulse width is $700 \mathrm{fs}$ whether using the SLM or bypassing it with a flat mirror. For broadband applications, one can use a metal coated 


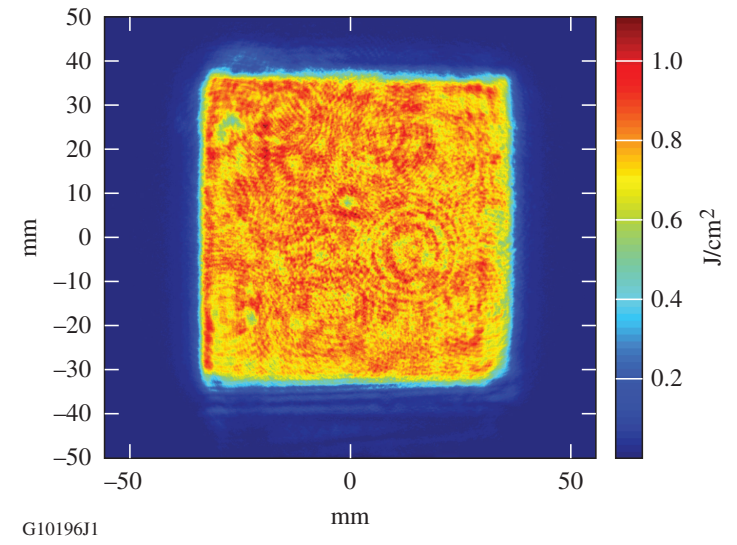

Figure 10: (Color online) Beam profile of the disk-amplifier output near an image plane. The energy in the beam is $39 \mathrm{~J}$ and the full-width-at-half-maximum is $70 \mathrm{~mm}$.

SLM with reduced reflectivity (Hamamatsu X10468-07). As the phase response is slightly different at different wavelengths, the diffraction efficiency cannot be maintained at a single value over a broad bandwidth. This results in the blurring of beam shaping and a reduced contrast. Fig. 2(b) of [13] indicates 0.007 $\mathrm{rad} / \mathrm{nm}$ change at $\pi$ phase shift for this type of device. Allowing $0.3 \mathrm{rad}$ tolerance in beam shaping carrier amplitude, the bandwidth of the beam is limited to about $45 \mathrm{~nm}$.

The dispersion of the SLM can be measured using white light interferometry [14]. The total dispersion of the SLM consists of the dispersion in the cover glass and the Gires-Tournois (GT) interference from the liquid crystal layer and its surrounding structure such as the semi-transparent conducting layer and alignment layers. The double passing of the 3 - $\mathrm{mm}$ thick coverglass introduces $0.7 \mathrm{rad}$ of spectral phase from 750 to $1000 \mathrm{~nm}$ after removing the quadratic term. The spectral phase of the GT interference in the broadband SLM sample (X10468-07) has modulation of $0.05 \mathrm{rad}$ in $\mathrm{p}-\mathrm{v}$ with $24 \mathrm{~nm}$ periodicity in offstate. The spectral and temporal responses of similar LCOSSLM's in on-state were characterized in [15] using different methods. The p-v of the GT modulation there was shown to be $0.15 \mathrm{rad}$ with $45-\mathrm{nm}$ periodicity at the full gray level. This level of modulation creates small pre- and post-pulses only visible in logarithmic scale and does not affect the main pulse. More importantly, the temporal diffraction from the steep edge of the spectrum is more dominant than the weak pre/post-pulses from the GT etalon effect because the broadband spectrum usually has a super-Gaussian like shape.

\section{Conclusion}

The previous carrier-beam-shaping method using an SLM was improved in both open-loop and closed-loop algorithms. The improvements to the open-loop algorithm was based on using a measured transmission function rather than a theoretical function. The measured function can still be expressed as a simple analytic function with three parameters specific to the device and the carrier type. A new search method dubbed as the constant maximum-derivative Newton method improves the closed-loop algorithm in terms of convergence stability and speed. The details of both wavefront and fluence shaping closedloop algorithms were summarized in Eqs. (16) and (17). A new method of carrier-beam-shaping method using a sawtooth carrier and the first order diffraction as the main beam was explored. It has a fail-safe feature and higher beam contrast, which might be useful in some applications.

The beam-shaping system was implemented in a multiterawatt OPCPA laser. The gain precompensation of a 200-mJ, 2.5-ns OPCPA laser beam improves the beam uniformity of the next stage amplification. The objective map for the precompensation is designed based on pre and post amplification beam profiles. The improved uniformity in the precompensated amplified beam is striking in that it is better than the unamplified OPCPA input beam. The improved uniformity in the amplified beam through the rod amplifier with the help of beam shaping allows for a safer energy ramp in the next-stage boost amplifier and a better protection of the gratings in the pulse compressor. The best beam shape can be maintained thanks to the dynamic adjustability of the beam shaper regardless of the small daily changes in the OPCPA beam profile and the gain response in the rod amplifier.

Other important issues were also addressed in applying the SLM-based beam shaping system to a high-energy laser. The device can be run safely below the damage threshold even at full OPCPA energy by utilizing the entire available SLM area. This is done by anamorphically imaging a square input beam onto the rectangular area of the SLM. The prepulse coming from the SLM's front surface, which adversely affects a solid-target experiment, was eliminated by using a customized wedged cover glass.

With future improvements in the damage threshold and the bandwidth of a liquid crystal device that can be used with the carrier-beam-shaping method, a beam-shaping system similar to the one described in this article may find a broader application in improving laser performance.

This material is based upon work supported by the Department of Energy National Nuclear Security Administration under Award Number DE-NA0001944, the University of Rochester, and the New York State Energy Research and Development Authority. The support of DOE does not constitute an endorsement by DOE of the views expressed in this paper.

\section{References}

[1] V. Bagnoud, J. D. Zuegel, Independent phase and amplitude control of a laser beam by use of a single-phase-only spatial light modulator, Optics Letters 29 (2004) 295-297.

[2] S. Bahk, E. Fess, B. E. Kruschwitz, J. D. Zuegel, A high-resolution, adaptive beam-shaping system for high-power lasers, Optics Express 18 (2010) 9151-9163.

[3] M. Barczys, S.-W. Bahk, M. Spilatro, D. Coppenbarger, E. Hill, T. H. Hinterman, R. W. Kidder, J. Puth, T. Touris, J. D. Zuegel, Deployment of a spatial light modulator-based beam-shaping system on the omega ep laser, Proc. SPIE 8602 (2013) 86020F-86020F-12.

[4] C. Dorrer, J. D. Zuegel, Design and analysis of binary beam shapers using error diffusion, Journal of the Optical Society of America B 24 (2007) 1268-1275.

[5] J. Heebner, M. Borden, P. Miller, S. Hunter, K. Christensen, M. Scanlan, C. Haynam, P. Wegner, M. Hermann, G. Brunton, E. Tse, A. Awwal, 
N. Wong, L. Seppala, M. Franks, E. Marley, K. Williams, T. Budge, M. Henesian, C. Stolz, T. Suratwala, M. Monticelli, D. Walmer, S. Dixit, C. Widmayer, J. Wolfe, J. Bude, K. McCarty, J.-M. DiNicola, Programmable beam spatial shaping system for the national ignition facility, Proc. SPIE 7916 (2011) 79160H-79160H-6.

[6] S. Bahk, J. D. Zuegel, J. R. Fienup, C. C. Widmayer, J. Heebner, Spotshadowing optimization to mitigate damage growth in a high-energy-laser amplifier chain, Applied Optics 47 (2008) 6586-6593.

[7] M. J. Guardalben, L. J. Waxer, Improvements to long-pulse system performance and operational efficiency on omega ep, Proc. SPIE 7916 (2011) 79160G-79160G-10.

[8] T. Zhao, J. Yu, C. Li, K. Huang, Y. Ma, X. Tang, Z. Fan, Beam shaping and compensation for high-gain nd:glass amplification, Journal of Modern Optics 60 (2012) 109-115.

[9] S. Ngcobo, I. Litvin, L. Burger, A. Forbes, A digital laser for ondemand laser modes, Nature Communications (2013) 4:2289 doi: 10.1038/ncomms3289.

[10] V. Bagnoud, J. Puth, I. Begishev, M. Guardalben, J. D. Zuegel, N. Forget, C. L. Blanc, J. Bromage, A multiterawatt laser using a high-contrast, optical parametric chirped-pulse preamplifier, in: Conference on Lasers and Electro-Optics/Quantum Electronics and Laser Science and Photonic
Applications Systems Technologies, Optical Society of America, 2005, p. JFA1.

[11] C. Dorrer, I. A. Begishev, A. V. Okishev, J. D. Zuegel, High-contrast optical-parametric amplifier as a front end of high-power laser systems, Opt. Lett. 32 (2007) 2143-2145.

[12] C. A. Haynam, P. J. Wegner, J. M. Auerbach, M. W. Bowers, S. N. Dixit, G. V. Erbert, G. M. Heestand, M. A. Henesian, M. R. Hermann, K. S. Jancaitis, K. R. Manes, C. D. Marshall, N. C. Mehta, J. Menapace, E. Moses, J. R. Murray, M. C. Nostrand, C. D. Orth, R. Patterson, R. A. Sacks, M. J. Shaw, M. Spaeth, S. B. Sutton, W. H. Williams, C. C. Widmayer, R. K. White, S. T. Yang, B. M. V. Wonterghem, National ignition facility laser performance status, Applied Optics 46 (2007) 3276-3303.

[13] H. Huang, T. Inoue, H. Tanaka, Stabilized high-accuracy correction of ocular aberrations with liquid crystal on silicon spatial light modulator in adaptive optics retinal imaging system, Optics Express 19 (2011) 15026.

[14] S. Diddams, J.-C. Diels, Dispersion measurements with white-light interferometry, J. Opt. Soc. Am. B 13 (1996) 1120-1129.

[15] M. Bock, S. K. Das, R. Grunwald, S. Osten, P. Staudt, G. Stibenz, Spectral and temporal response of liquid-crystal-on-silicon spatial light modulators, Applied Physics Letters 92 (2008) 151105. 